\title{
Clasificación y mapeo automático de coberturas del suelo en imágenes satelitales utilizando Redes Neuronales Convolucionales
}

\author{
Classification and automatic mapping of land covers in satellite \\ images using Convolutional Neural Networks
}

\section{Classificação e mapeamento automático de coberturas do solo em imagens de satélite usando redes neurais convolucionais}

\author{
Arnol S Suárez $\mathrm{L}^{1 *}$; Andrés F Jiménez $\mathrm{L}^{2 *}$; Mauricio Castro-Franco ${ }^{3}$; Angel Cruz-Roa ${ }^{4}$
}

1 Ingeniero electrónico

2 Ingeniero electrónico, MSc, estudiante de doctorado en ingeniería - mecánica mecatrónica, Grupo de investigación Un-Robot, UNAL, Bogotá, Colombia

3 Ingeniero Agrónomo, Dr.Sc, estudiante de Posdoctorado en Ciencias Agrarias Consejo Nacional de Investigaciones Científicas y Técnicas CONICET, Buenos Aires, Argentina.

4 Ingeniero de Sistemas, MSc, PhD, grupo de Investigación GITECX, Facultad de Ciencias Básicas e Ingeniería, Universidad de los Llanos, Villavicencio, Colombia.

* Grupo de investigación Macrypt, Facultad de Ciencias Básicas e Ingeniería, Universidad de los Llanos, Villavicencio, Colombia

Email: aacruz@unillanos.edu.co

\section{Resumen}

La clasificación de cobertura del suelo es importante para estudios de cambio climático y monitoreo de servicios ecosistémicos. Los métodos convencionales de clasificación de coberturas se realizan mediante la interpretación visual de imágenes satelitales, lo cual es costoso, dispendioso e impreciso. Implementar métodos computacionales permite generar clasificación de coberturas en imágenes satelitales de manera automática, rápida, precisa y económica. Particularmente, los métodos de aprendizaje automático son técnicas computacionales promisorias para la estimación de cambios de cobertura del suelo. En este trabajo se presenta un método de aprendizaje automático basado en redes neuronales convolucionales de arquitectura tipo ConvNet para la clasificación automática de coberturas del suelo a partir de imágenes Landsat 5 TM. La ConvNet fue entrenada a partir de las anotaciones manuales por medio de interpretación visual sobre las imágenes satelitales con las que los expertos generaron el mapa de cobertura del parque nacional el Tuparro, de los Parques Nacionales Naturales de Colombia. El modelo de validación se realizó con datos de los mapas de coberturas del Amazonas colombiano realizado por el Sistema de Información Ambiental de Colombia. Los resultados obtenidos de la diagonal de la matriz de confusión de la exactitud promedio fue de $83.27 \%$ en entrenamiento y $91.02 \%$ en validación; para la clasificación en parches entre Bosques, áreas con vegetación herbácea y/o arbustiva, áreas abiertas sin o con poca vegetación y aguas continentales.

Palabras Clave: Aprendizaje automático, coberturas de suelo, parques naturales, redes neuronales convolucionales, teledetección. 


\begin{abstract}
Land cover classification is important for studies of climate change and monitoring of ecosystem services. Conventional coverage classification methods are performed by the visual interpretation of satellite imagery, which is expensive and inaccurate. Implementing computational methods could generate procedures to classify coverage in satellite images automatically, quickly, accurately and economically. Particularly, automatic learning methods are promising computational methods for estimating soil cover changes. In this work we present an automatic learning method based on convolutional neural networks of ConvNet type architecture for the automatic classification of soil coverings from Landsat 5 TM images. The ConvNet was trained from the manual annotations by means of visual interpretation on the satellite images with which the experts generated the map of Tuparro national park, of National Natural Park of Colombia. The validation model was performed with data from the Colombian Amazon cover maps made by the Colombian Environmental Information System. The results obtained from the diagonal of the confusion matrix of the average accuracy were $83.27 \%$ in training and $91.02 \%$ in validation; for the classification in patches between forests, areas with herbaceous and / or shrub vegetation, open areas with or without vegetation and Inland waters.
\end{abstract}

Keywords: Automatic learning, land cover, natural parks, convolutional neural networks, remote sensing.

\begin{abstract}
Resumo
A classificação da cobertura da terra é importante para estudos de mudanças climáticas e monitoramento dos serviços dos ecossistemas. Os métodos convencionais de classificação de cobertura são feitos através da interpretação visual de imagens de satélite, que é caro, dispendioso e impreciso. Implementar métodos computacionais poderia gerar procedimentos de classificação de cobertura em imagenes de satélite de forma automática, rápida, precisa e econômica. Particularmente, métodos de aprendizado de máquina são promissores métodos computacionais para estimar a cobertura do solo mudanças. Neste artigo apresentamos um método de aprendizado de máquina baseado em convolutional neural tipo ConvNet rede de arquitetura para a classificação automática de cobertura do solo a partir de Landsat 5 imagens TM. O ConvNet foi treinado desde anotações manuais através da interpretação visual das imagens de satélite que os especialistas geraram o mapa de cobertura do Parque Nacional Tuparro, Colômbia Parque Nacional Natural. A validação do modelo foi realizada com cobertura de mapa de dados da Amazônia colombiana pelo Sistema de Informação Ambiental da Colômbia. Os resultados da diagonal da matriz de confusão da precisão média foi de 83,27\% e Formação e 91,02\% na validação; para a classificação em manchas entre florestas, áreas com vegetação herbácea e / ou arbusto, áreas abertas com pouca ou nenhuma vegetação águas interiores.
\end{abstract}

Palavras-chave: Aprendizagem de máquina, cobertura do solo, parques naturais, rede neural convolutional, sensoriamento remoto.

\section{Introducción}

La cobertura del suelo hace referencia a la información del material físico en la superficie de la tierra y el uso del suelo se asocia a las modificaciones hechas sobre esta cobertura por el hombre. La detección de cambios de cobertura o uso, es el proceso de identificar los cambios en un área o fenómeno ambiental mediante la observación en diferentes momentos de tiempo (Berberoglu et al., 2009). La clasificación de cobertura del suelo es importante para estudios de cambio climático y monitoreo de servicios ecosistémicos (Rodriguez et al., 2012) además de ser vital en los estudios del cambio climático global, fenómenos naturales, monitoreo y cuantificación de recursos, entre otros (Backoulou et al., 2015; Bokusheva et al., 2016; Eisavi et al., 2015).

La clasificación de coberturas del suelo, consiste en estimar los tipos de coberturas en un área determinada ya sea usando observación visual o métodos computacionales. La clasificación de coberturas, es aplicada a cartografía (Eisavi et al., 2015), monitoreo de recursos naturales (Chen et al., 2006), cambios en la biodiversidad de un área analizando los índices de vegetación (Bokusheva et al., 2016) y monitoreo de cultivos en busca de infecciones (Otukei et al., 2010).

El procesamiento de información adquirida mediante teledetección se ha utilizado para el estudio de las coberturas del suelo, en especial las imágenes satelitales (Shao et al., 2012). La teledetección usa la reflectancia de la tierra para obtener información de los materiales y características de su superficie (Warner et al., 2009). Gracias a la resolución espacial y espectral de las imágenes adquiridas, se puede cubrir áreas extensas y gracias a las bandas que poseen de las regiones del espectro electromagnético (usualmente de 2 a 10 bandas espectrales - multiespectrales), se pueden estudiar aspectos fenológicos de la cobertura terrestre. Las imágenes de satélite se estudian como la representación visual de la reflectancia de la tierra adquirida por el sensor particular, en donde se han desarrollado procedimientos de representación de esta información, como las firmas espectrales y los índices de vege- 
tación. Las principales regiones espectrales utilizadas para el estudio de la superficie de la tierra son: visible (Azul, Verde, Rojo), infrarrojo cercano, infrarrojo de onda corta, infrarrojo de onda media, infrarrojo de onda larga o térmico, microondas y radar (Schowengerdt, 2007).

La clasificación de tipos de cobertura del suelo es una tarea que demanda capacidad de cómputo, debido a la gran cantidad de datos que se tiene que procesar, por lo cual, se establece la necesidad de aplicar métodos computacionales suficientemente rápidos y efectivos. El aprendizaje computacional es una rama de la inteligencia artificial cuyo objetivo es desarrollar técnicas, métodos y algoritmos que permiten que los computadores aprendan a reconocer patrones a partir de datos de un modelo de inferencia con propósitos predictivos (Alpaydin, 2014). Estos métodos son cada vez más utilizados en la clasificación de imágenes de teledetección en aplicaciones de monitoreo de recursos naturales (Mishra, 2015).

Entre los tipos de algoritmos de aprendizaje automático se destacan dos, el aprendizaje supervisado y el aprendizaje no supervisado. El aprendizaje supervisado ocurre cuando se proporciona al modelo de entrenamiento datos conocidos, es decir cuando se sabe a qué clase pertenece cada uno. De hecho, estos algoritmos han sido ampliamente utilizados en la clasificación de coberturas del suelo como es el caso de las máquinas de soporte vectorial (Wang, 2013). En los algoritmos de aprendizaje no supervisado el modelo de entrenamiento está formado por entradas y no se tiene información a qué clase pertenecen los datos, por lo tanto, su objetivo es agrupar los datos por características o patrones similares en un número definido de clases.

Con el fin de disminuir el costo y el error humano, se han investigado métodos computacionales para mejorar el rendimiento y la precisión de la clasificación de coberturas del suelo (Thonfeld et al., 2011) Previamente se han empleado entre otros métodos de aprendizaje computacional: los árboles de decisión (Rodríguez-Galiano et al., 2012), las cadenas de Markov (Halmy et al., 2015), la diferenciación de imágenes y cambio del vector de análisis (Berberoglu et al., 2009), determinados a través de la matriz de transición de los mapas de cobertura (Romero-Ruiz, 2012), el análisis de componentes principales (ACP) (Romero et al., 2012), las firmas espectrales y los índices de vegetación (Backoulou et al., 2015).

El método de clasificación automática de Máxima Verosimilitud (en inglés, Maximum Likelihood - ML) ha sido uno de los más usados en los últimos 40 años en coberturas de la tierra (Warner et al., 2009), (Otukei et al., 2010). En la actualidad, los algoritmos de aprendizaje computacional como las máquinas de soporte vectorial (en inglés, Support Vector Machine - SVM) y Bosques Aleatorios (en inglés, Random Forest - RF), han sido ampliamente utilizados para la clasificación automática con resultados de precisión general de $86.5 \%$ (Eisavi et al., 2015) y $95.10 \%$ de precisión de clasificación (Liu et al., 2013). Entre las medidas de desempeño y evaluación en la clasificación de coberturas y uso del suelo se utiliza el coeficiente de Kappa de Cohen (Wang et al., 2016) y en algoritmos de aprendizaje computacional se utiliza el promedio de exactitud sobre la matriz de confusión de los datos de salida (Al Obeidat, 2015).

Las Redes Neuronales Convolucionales (en inglés Convolutional Neural Networks - CNN), son un método bio-inspirado de aprendizaje computacional, y han demostrado ser eficientes en diferentes áreas, como en el reconocimiento de voz (Huang, Li, y Gong, 2015), así como en clasificación automática de imágenes de personas por género, ropa inferior y superior (Perlin et al., 2015), aplicaciones en imágenes médicas como en la detección de cáncer de mama invasivo (Wang et al., 2014), (Cruz-Roa et al., 2014) y diferenciación de tumor meduloblastoma (Cruz-Roa et al., 2015).

En Colombia, la investigación de métodos automáticos para la creación de mapas de coberturas de suelo es escasa; el alto costo y el tiempo invertido en su creación están vinculados a la calidad y disposición de imágenes de productos de sensores remotos satelitales, y al juicio del profesional a cargo de la realización del mapa (IDEAM, IGAC, \& CORMAGDALENA, 2008). Como ejemplo de estos procedimientos de gran esfuerzo, se tiene la información de los mapas de Parques Naturales Nacionales (PNN) de Colombia (Acevedo, 2012) y del Sistema de Información Ambiental de Colombia (SIAC) (Axesnet S.a.S, 2012); que son utilizados en esta investigación.

Este trabajo presenta el desarrollo y evaluación de un método computacional basado en redes neuronales convolucionales para la clasificación de suelos con la definición de clases del segundo nivel: bosques, áreas con vegetación herbácea y/o arbustiva, áreas abiertas, sin o con poca vegetación y áreas húmedas continentales. Clases definidas por leyenda nacional de coberturas de la tierra metodología CORINE Land Cover, adaptada para Colombia, escala 1:100.000 (Ministerio del Medio Ambiente, 2010). 
El método propuesto empieza con la construcción de un conjunto de entrenamiento con el mapa de cobertura de suelo del parque nacional el Tuparro realizado por PNN, con el cual se entrenó una Red Neuronal Convolucional de arquitectura ConvNet, mientras que en el conjunto de validación se evaluó con los mapas de coberturas del Amazonas del 2007 realizado por el SIAC. De esta forma, se generaron predicciones de mapas de cobertura con el modelo entrenado y validado de la ConvNet.

El artículo está divido en secciones, en Materiales y Métodos se presenta la metodología de la técnica propuesta para la clasificación de las coberturas del suelo, así como el conjunto de datos generados para el entrenamiento y validación para la CNN, en Resultados se presentan los resultados obtenidos en gráficas y tablas, mientras que en la sección Discusión se analizan estos resultados obtenidos de los experimentos realizados. Finalmente, en la última sección Conclusiones se dan a conocer las principales conclusiones del método de clasificación y mapeo automático de coberturas del suelo en imágenes satelitales utilizando redes neuronales convolucionales.

\section{Materiales y métodos}

\section{Método computacional para la clasificación de coberturas del suelo a partir de imágenes satelitales.}

La implementación de las CNN en la tarea de clasificación de coberturas del suelo presenta un reto completamente nuevo y diferente a los trabajos anteriormente mencionados, con pocos o ningún trabajo realizado en el área de coberturas de suelos, por lo que en esta sección se describirá la metodología utilizada para la implementación de las $\mathrm{CNN}$, desde la generación del conjunto de entrenamiento y validación hasta la implementación y comparación con métodos tradicionales actuales.

Para el desarrollo de este trabajo se realizó el procedimiento explicado en la Figura 1. Inicialmente se diseñó e implementó un algoritmo de preprocesamiento de la información espectral de imágenes satelitales Landsat 5 TM que permitiera obtener los mapas útiles como conjunto de entrenamiento y validación, teniendo en cuenta los aspectos relacionados con la georreferenciación y la corrección de defectos tanto en imágenes como en mapas de insumo. Luego se desarrolló la etapa de extracción de muestras de regiones cuadradas de terreno de las clases sobre las imágenes satelitales para la generación del conjunto de entrenamiento y validación. Posteriormente, se realizó el algoritmo de CNN para los procesos de entrenamiento y validación para poder entrenar el modelo final. Finalmente se realizaron predicciones de mapas de cobertura con el modelo de la CNN y se calculó la similitud entre los mapas reales anotados por expertos y los generados automáticamente.

\section{Selección y Preprocesamiento}

Se utilizó información de cobertura correspondiente a mapas del parque el Tuparro realizado el 2007 por Parques Nacionales Naturales de Colombia (PNN)

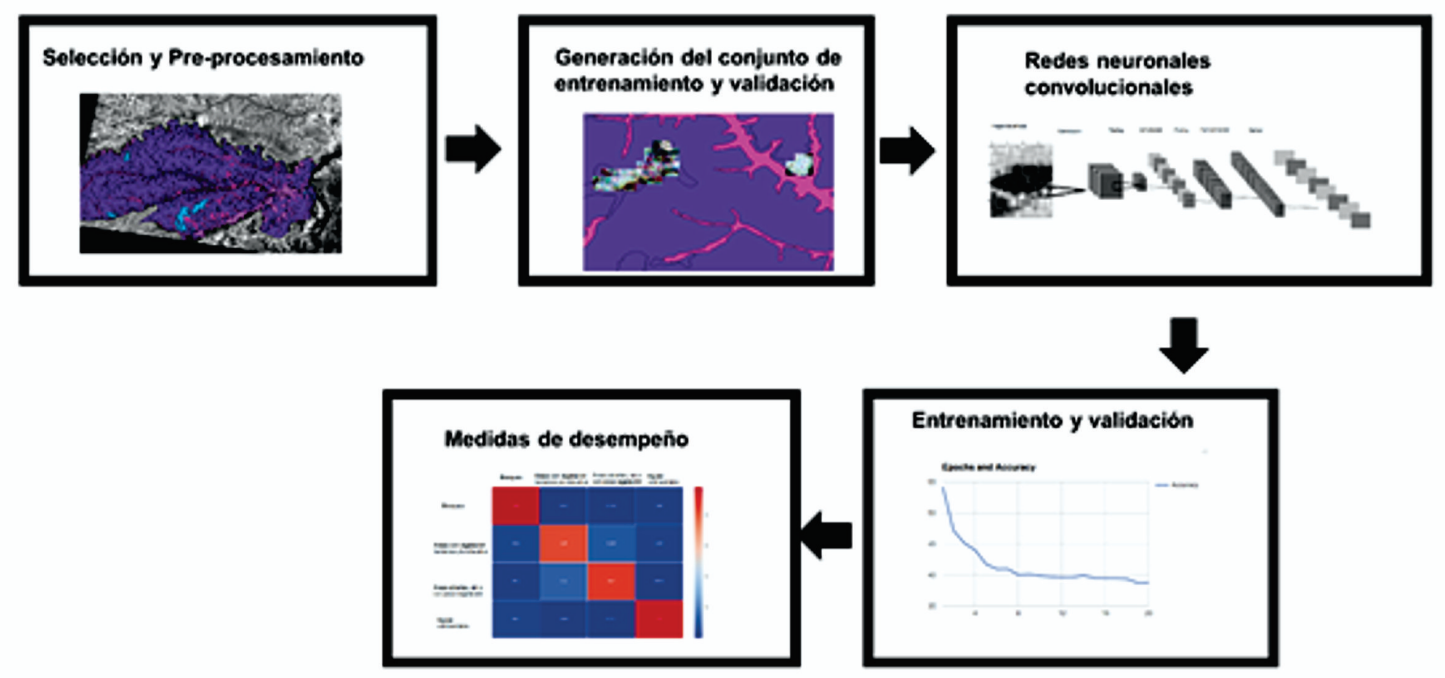

Figura 1. Procedimiento para la clasificación de coberturas de suelo. Fuente: Autores. 
y los mapas de coberturas del Amazonas del 2007, realizados por el Sistema de Información Ambiental de Colombia (SIAC). Los mapas utilizados en este proyecto se pueden observar en la Figura 2. Se descargaron las imágenes satelitales utilizadas para realizar estos mapas de cobertura compuestas por imágenes Landsat 456 21/01/2008, las cuales se utilizaron como insumo para el mapa del parque el Tuparro. De la misma manera para el mapa de cobertura del Amazonas del 2007, se descargaron las imágenes Landsat 658 2008/04/08, que cuentan con cobertura de áreas de interés para el estudio. Para garantizar que la información corresponda espacialmente, se homogeniza el sistema de referencia de coordenadas (SRC) de imágenes y mapas a Magna-Sirgas de Colombia. El mapa del parque el Tuparro y la imagen satelital se le asignó el SRC Magna-Sirgas 3118 y a las demás imágenes y mapas se le asignó el SRC Magna-Sirgas 3117. Las imágenes Landsat 658 2008/04/08 y Landsat 456 21/01/2008 contenían píxeles con gran cantidad de nubosidad, lo cual introducía errores a la hora de procesar la imagen. Por lo tanto, se utilizó el programa QuantumGIS ${ }^{1}$ para la eliminación de las áreas que coincidían con las que corresponden con estos comportamientos. Con la ayuda del programa $\mathrm{ENVI}^{2}$ se realizó la corrección atmosférica de las imágenes utilizadas en este trabajo y por último se calculó la media y desviación estándar de los datos de entrenamiento. Esta información es necesaria para los procedimientos de normalización de los datos.

\section{Extracción de muestras (regiones cuadradas o parches)}

El conjunto de entrenamiento se generó utilizando el mapa del parque el Tuparro con un área de total de $5,564 \mathrm{~km}^{2}$ del cual se utilizaron $4,299 \mathrm{~km}^{2}$ para realizar la extracción de parches (muestras de las clases) de 20 × 20 píxeles. Para realizar la extracción de los parches se realizó un algoritmo en el lenguaje de programación Python, el cual se encarga de leer las imágenes satelitales y los mapas de cobertura. Los mapas se separaron por clases y se desarrolló el procedimiento de extracción de parches de 20 x 20 x 7 píxeles (Ancho, Alto, Bandas), con pasos (strides) de 20 píxeles menos el solapamiento de $50 \%$, se verificaba que los parches cuadrados tuvieran al menos un $80 \%$ de su área perteneciente a una clase, de acuerdo con las regiones anotadas por los expertos en el conjunto de entrenamiento, para que el parche sea

\footnotetext{
http://www.qgis.org/

http://www.harrisgeospatial.com/ProductsandSolutions/ GeospatialProducts/ENVI.aspx
}

asociado a la clase. Para las clases Áreas abiertas, sin o con poca vegetación y Áreas húmedas continentales se les hizo un sobre-muestreo y solapamiento del $80 \%$ entre muestras, debido a que las áreas de interés eran muy pequeñas o delgadas (cuando correspondían a ríos). A cada parche de las clases Áreas abiertas, sin o con poca vegetación y Áreas húmedas continentales se le realizaron rotaciones de 90 grados e inversiones para generar 8 parches adicionales por clase. A la clase Áreas con vegetación herbácea o arbustiva, no se le realizó solapamiento para la extracción de muestras, debido a que era la clase que tenía mayor cantidad de área. Se generó un total de 56,935 parches, correspondientes a $683.22 \mathrm{~km}^{2}$; el número de parches por clases se aprecia en la Tabla 1. Los parches generados no son diferenciables a simple vista ya que las imágenes satelitales de cada banda están representadas en escala de grises, como se observa en la Figura 3.

Tabla 1. Número de parches según clases de entrenamiento.

\begin{tabular}{|l|c|c|}
\hline \multicolumn{3}{|c|}{ Balance de clases } \\
\hline \multicolumn{1}{|c|}{ Clases } & $\begin{array}{c}\text { Número de } \\
\text { parches }\end{array}$ & $\begin{array}{c}\text { Área total en } \\
\text { parches }\left(\mathbf{m}^{2}\right)\end{array}$ \\
\hline Bosques & 17,608 & $211,296,000$ \\
\hline $\begin{array}{l}\text { Áreas con vegetación } \\
\text { herbácea y/o arbustiva }\end{array}$ & 18,303 & $219,636,000$ \\
\hline $\begin{array}{l}\text { Áreas abiertas, sin o } \\
\text { con poca vegetación }\end{array}$ & 12,840 & $633,264,000$ \\
\hline $\begin{array}{l}\text { Areas húmedas } \\
\text { continentales }\end{array}$ & 8,184 & $154,080,000$ \\
\hline
\end{tabular}

Fuente: Autores.

Los parches generados de cada clase contienen las siete bandas de la imagen original como se puede apreciar en la Figura 4. Los parches sin información en alguna de sus bandas son desechados. El conjunto de entrenamiento para la CNN necesita estar balanceado (i.e. similar proporción de parches de ejemplo por clase) para que el modelo predictivo no se incline a la clase con el mayor número de muestras (sobreentrenamiento). Por lo tanto, se descartaron datos de algunas de las clases para obtener una cantidad de parches por clase más homogénea, tal como se observa en la Tabla 2.

La información que se utilizó para elaborar el conjunto de validación corresponde a áreas de interés del mapa de cobertura del Amazonas del 2007 con una extensión total de 7,243 km²; se corrió el algoritmo para la extracción de parches generando un total de 21,584 parches, distribuidos como se muestra en la Tabla 3. 


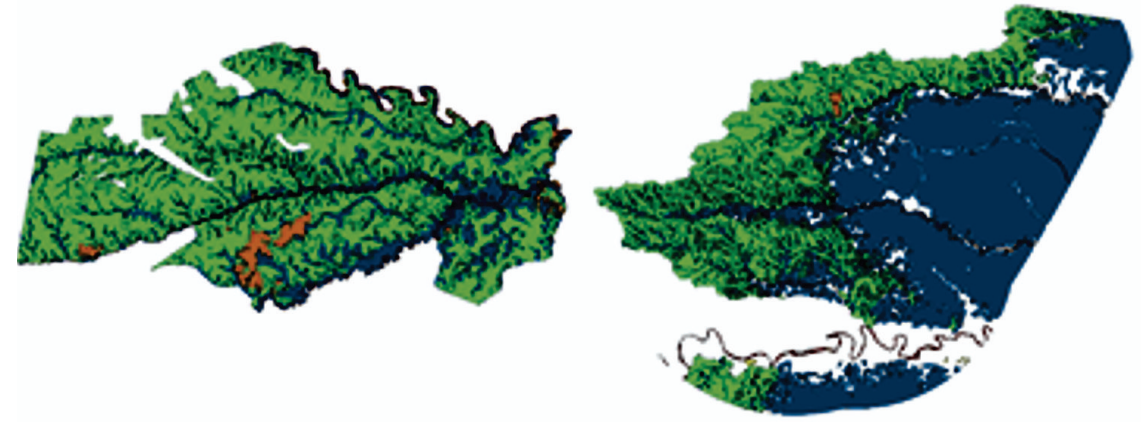

Clases

Bosques

Áreas con vegetación herbácea y/o arbustiva

Áreas abiertas, sin 0 con poca vegetación

Áreas húmedas continentales

Figura 2. Mapas utilizados en el estudio. Izquierda: área de interés del mapa de coberturas del Amazonas 2007. Derecha: mapa del Parque Nacional el Tuparro.

Fuente: (Acevedo, 2012), (Axesnet S.a.S, 2012)

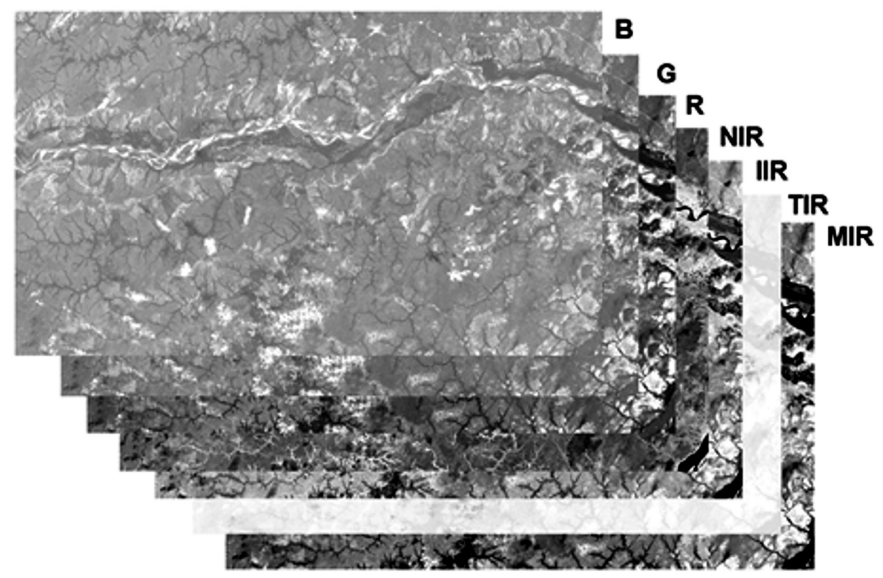

Figura 3. Imagen del satelite Landsat TM 5. B: Azul, G: Verde, R: Rojo, NIR: infrarojo cercano, IIR: Infrarojo intermedio, TIR: Infrarojo termico y MIR: Infrarojo medio.

Fuente: Autores.

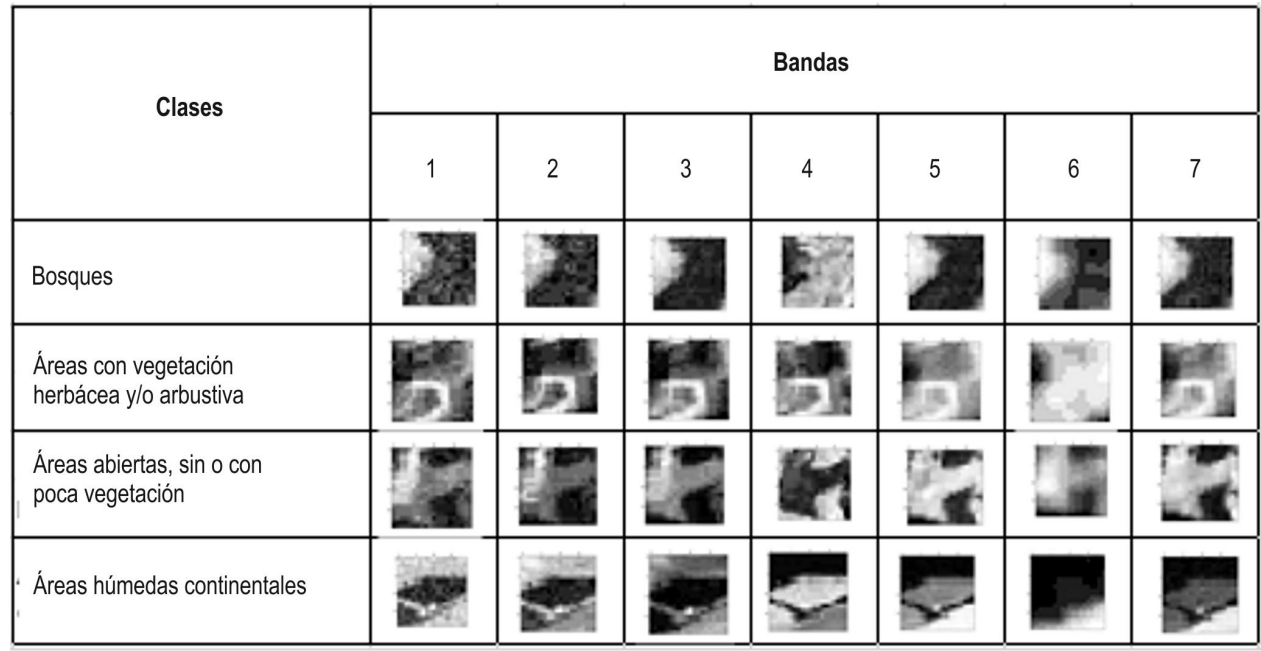

Figura 4. Ejemplo de parches por clase y sus bandas.

Fuente: Autores. 
Note que el conjunto de validación no tiene que ser balanceado. Se calcula la media y desviación estándar de todos los parches de entrenamiento para realizar una normalización puntual estándar sobre todos los parches tanto de entrenamiento como de validación.

Tabla 2. Número de parches despues de hacer el balanceo de clases.

\begin{tabular}{|l|c|c|}
\hline \multicolumn{3}{|c|}{ Balance de clases } \\
\hline \multicolumn{1}{|c|}{ Clases } & $\begin{array}{c}\text { Número de } \\
\text { parches }\end{array}$ & $\begin{array}{c}\text { Área total en } \\
\left.\text { parches } \mathbf{( m}^{2}\right)\end{array}$ \\
\hline Bosques & 10,000 & $120,000,000$ \\
\hline $\begin{array}{l}\text { Áreas con vegetación } \\
\text { herbácea y/o arbustiva }\end{array}$ & 10,000 & $120,000,000$ \\
\hline $\begin{array}{l}\text { Áreas abiertas, sin o con } \\
\text { poca vegetación }\end{array}$ & 10,000 & $120,000,000$ \\
\hline $\begin{array}{l}\text { Areas húmedas } \\
\text { continentales }\end{array}$ & 8,184 & $98,208,000$ \\
\hline
\end{tabular}

Fuente: Autores.

Tabla 3. Número de parches usados en la validación.

\begin{tabular}{|l|c|c|}
\hline \multicolumn{3}{|c|}{ Balance de clases } \\
\hline \multicolumn{1}{|c|}{ Clases } & $\begin{array}{c}\text { Número de } \\
\text { parches }\end{array}$ & $\begin{array}{c}\text { Área total en } \\
\left.\text { parches } \mathbf{( m}^{2}\right)\end{array}$ \\
\hline Bosques & 12,927 & $155,124,000$ \\
\hline $\begin{array}{l}\text { Áreas con vegetación } \\
\text { herbácea y/o arbustiva }\end{array}$ & 8,041 & $96,492,000$ \\
\hline $\begin{array}{l}\text { Áreas abiertas, sin o con } \\
\text { poca vegetación }\end{array}$ & 538 & $6,456,000$ \\
\hline $\begin{array}{l}\text { Áreas húmedas } \\
\text { continentales }\end{array}$ & 78 & 936,000 \\
\hline
\end{tabular}

Fuente: Autores.

\section{Red neuronal convolucional}

Las redes neuronales convolucionales son el estado del arte en la clasificación automática de imágenes naturales (Krizhevsky et al., 2012). Las CNN tienen una fase de extracción de características y de clasificación. La extracción de características consta de una capa de convolución de la imagen de entrada con el filtro (kernel) con un número determinado de neuronas y una capa de sub-muestreo o pooling, en la cual se reduce la salida de la convolución. Posteriormente continúa una etapa de reducción de dimensionalidad y selección de características relevantes por medio de la capa de Fully-connected, y finalmente el modelo entrenado clasifica a qué clase pertenece una imagen en particular. Durante el proceso de entrenamiento, se utilizó el algoritmo de propagación hacia atrás (backpropa- gation) usando el gradiente estocástico con la CNN, el cual ajusta los pesos de los filtros y reduce el error de clasificación.

Las CNN requieren de conjuntos de datos de entrenamiento grandes, con el fin de ser más robustas a la hora de clasificar automáticamente. También es necesario que los datos de las clases estén balanceados si es un problema de múltiples clases. Tensorflow ${ }^{3}$ es una librería de código abierto de Google que fue seleccionada como herramienta de trabajo para elaborar el algoritmo de aprendizaje automático de CNN de arquitectura ConvNet con el fin de entrenar un modelo para la clasificación de coberturas. El algoritmo cuenta con dos capas de convolución y pooling. La primera capa realiza la convolución entre la imagen de entrada y el filtro (kernel) donde la imagen de entrada es de $20 \times 20 \times 7$, donde siete es el número de bandas de la imagen satelital, el filtro de $5 \times 5 \times 7 @$ C1 donde C1 es el número de neuronas por capa. A continuación, se realiza la etapa de pooling que para este caso corresponde a la función de Max-pooling, que hace un submuestreo eligiendo los valores máximos de la imagen en una ventana de $2 \times 2$. Se realiza una vez más las etapas de convolución y pooling, esta vez la convolución se realiza con los datos de la primera convolución y pooling de tamaño $8 \times 8 \times 7 @$ C1 y un segundo filtro kernel de $5 \times 5 \times \mathrm{C} 1 @ \mathrm{C} 2$ donde $\mathrm{C} 2$ es el número de neuronas de la segunda capa, otra capa de Max-pooling y por último se aplica la capa de neuronas totalmente conectadas (en inglés - fully connected) que serían los datos que entran en el clasificador. Los parámetros definidos para entrenar la $\mathrm{CNN}$ se pueden ver en la Tabla 4.

Tabla 4. Parámetros de entrenamiento Red Neuronal Convolucional.

\begin{tabular}{|l|c|}
\hline \multicolumn{1}{|c|}{ Tasa de aprendizaje } & $\mathbf{1 e}^{-5}$ \\
\hline Decaimiento de la tasa de aprendizaje & 0.9 \\
\hline Dropout & 0.5 \\
\hline Tamaño de lote & 569 \\
\hline Número de capas de convolución & 2 \\
\hline Neuronas en la primera capa & 128 \\
\hline Neuronas segunda capa & 256 \\
\hline Capa totalmente conectada & 1,024 \\
\hline
\end{tabular}

Fuente: Autores.

3 https://www.tensorflow.org/ 


\section{Entrenamiento}

Para realizar el entrenamiento de la CNN se desarrolló un módulo en Python que lee los conjuntos de datos, un $10 \%$ para probar el rendimiento de la CNN en entrenamiento y el resto para entrenar el modelo. Devuelve un objeto con atributos (imágenes y etiquetas) con un método que retorna un lote de estos parámetros, los cuales entrarán a la $\mathrm{CNN}$; una vez completada un ciclo o época, los datos se barajan y vuelven a pasar por la CNN hasta completar el número de once épocas. La primera fase de entrenamiento se realizó a lo largo de once épocas, una época es cuando todos los datos de entrenamiento pasan por la CNN, en la época número once se realizó una prueba de rendimiento sobre los datos de entrenamiento y validación sobre sus matrices de confusión como se puede apreciar en la Figura 5.
Se continuó entrenando el modelo hasta llegar a la época 27, donde se alcanzaron los datos con mejor desempeño de entrenamiento y validación como se muestra en la Figura 6. Al no encontrar mejora en los resultados obtenidos se detiene el procesamiento, observando una disminución en el error cuadrático medio (EMC) de la clasificación conforme transcurre cada época, como se aprecia en la Figura 7.

\section{Clasificación vista al vuelo}

Con el modelo de la CNN entrenado se realizó la predicción a vista de vuelo de los mapas de coberturas de las imágenes del Amazonas 2007. Se recorría la imagen cada 10 píxeles y se corría el modelo de la CNN sobre una porción de la imagen devolviendo las coordinas del punto y la predicción del modelo. Con las coordenadas y predicciones de las clases se implementó una interpolación de vecinos cercanos, así ma-

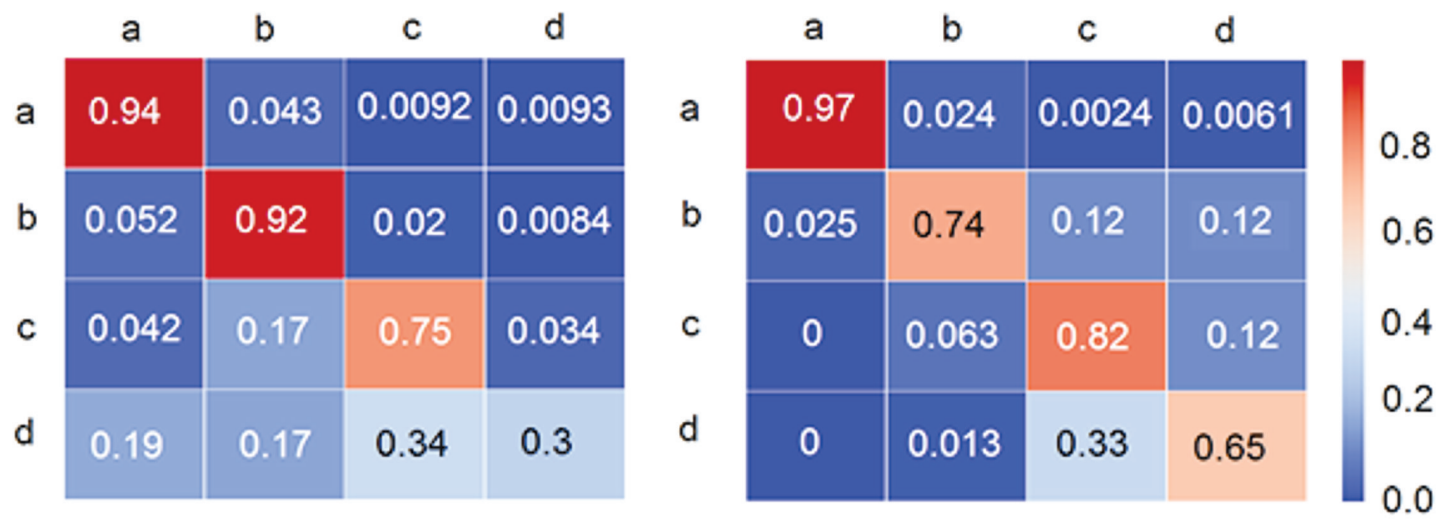

Figura 5. Matriz de confusión para las primeras 11 epocas. a: clase bosques, b: áreas con vegetación herbácea o arbustiva, c: áreas abiertas sin o con poca vegetación y d: áreas húmedas continentales [Izquierda Entrenamiento / Derecha Validación].

Fuente: Autores.

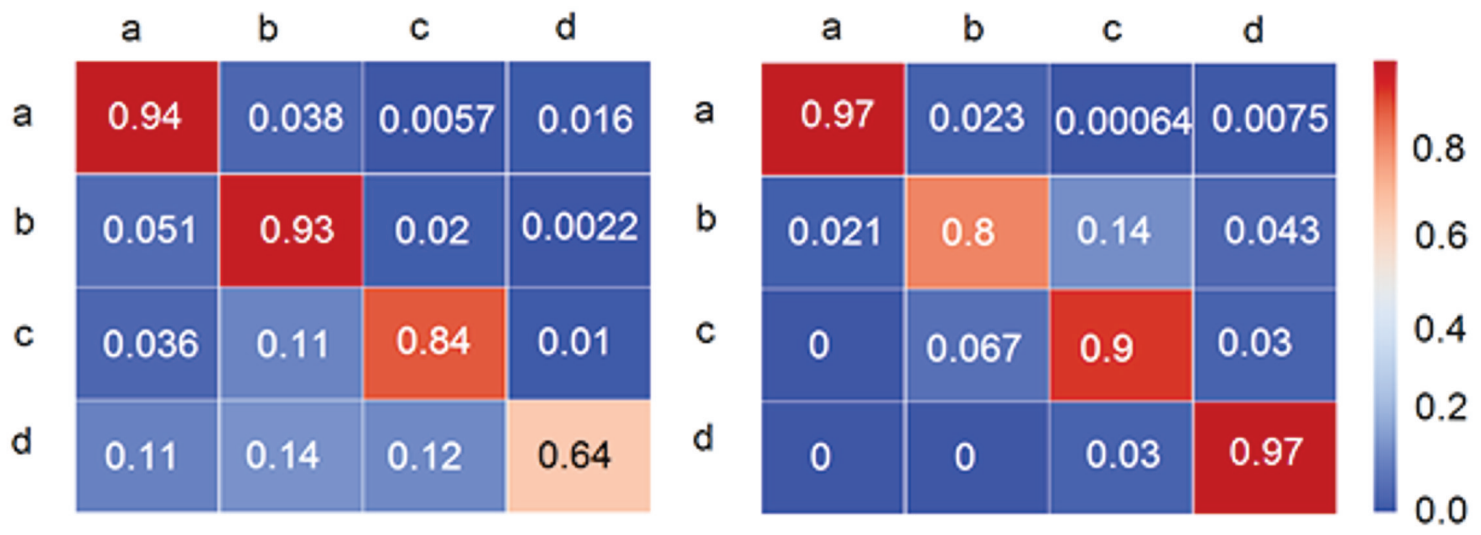

Figura 6. Matriz de confusión para la epoca 27. a: clase bosques, b: áreas con vegetación herbácea o arbustiva, c: áreas abiertas sin o con poca vegetación, d: áreas húmedas continentales [Izquierda Entrenamiento / Derecha Validación].

Fuente: Autores. 


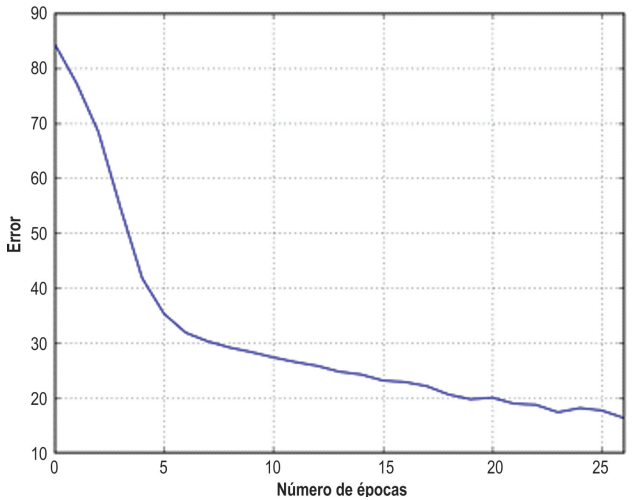

Figura 7. Reduccion del error cuadrático medio durante el entrenamiento.

Fuente: Autores.

peando el nuevo mapa predicho por la CNN como se muestra en la Figura 8. Con los mapas realizados con la CNN se calculó el coeficiente de Dice para medir la similitud de los mapas reales con los predichos como se puede ver en la Tabla 4. De esta forma, poder comparar la eficiencia del modelo de la CNN en clasificación y mapeo.

\section{Clasificación de cobertura del suelo usando firma espectral}

Las firmas espectrales han sido usadas para identificar un amplio tipo de coberturas (Martin et al., 1998). Con el fin de comparar los mapas predichos con la CNN usando métodos de clasificación tradicionales sin aprendizaje computacional, se realizó una clasificación usando las firmas espectrales de las clases usadas para entrenamiento. Se utilizó en algoritmo de distancia mínima, el cual clasifica a cada píxel y calcula la distancia mínima entre la firma espectral del píxel y cada clase, para asignar la pertenencia del píxel a la clase más cercana (Rujoiu-Mare et al., 2016). Se clasificó y mapeó un mapa con este procedimiento como se aprecia en la Figura 8.
Se utilizó la exactitud promedio sobre la matriz de confusión, la cual muestra el rendimiento por clases de los modelos entrenados previamente y también se determinó la desviación estándar de la diagonal de la matriz de confusión para apreciar el grado de dispersión de exactitud de la CNN entre las clases. Se utilizó el coeficiente de Dice para comparar la similitud de los mapas predichos con el modelo entrenado de la CNN y los mapas reales anotados por expertos, como se muestra en la Tabla 5.

\section{Resultados}

Se alcanzó un máximo de $83.27 \%$ de exactitud promedio sobre los datos de entrenamiento y un $91.02 \%$ en validación sobre sus matrices de confusión en parches como se muestra en la Figura 5. Se alcanzó un máximo de $88.7 \%$ de similitud al mapa real en la clasificación al vuelo y mapeo sobre la clase 1 , un $77.4 \%$ en la clase 2 , y unos valores mínimos en las clases 3 y 4 de los cuales se discutirán en la siguiente sección. Los coeficientes de Dice del mapa generado por la CNN son mayores a los coeficientes de Dice del mapa generado por las firmas espectrales y el algoritmo de distancia mínima como se aprecia en la Tabla 5.

\section{Discusión}

Los resultados finales obtenidos en rendimiento son altos para el procedimiento realizado con Redes Neuronales Convolucionales. La clasificación y mapeo de las clases 3 y 4 aunque funcionan en parches, en la aplicación práctica son muy difíciles de identificar debido a factores relacionados con la extensión espacial ocupada y la peculiaridad de sus formas, como lo es la clase 4 de áreas húmedas continentales, que hace referencia a los ríos. Las características estructurales y de forma de la clase 4, lo que hace difícil para nuestro modelo poder encontrar un río con un área de forma cuadrada de $600 \mathrm{~m}^{2}$, ya que generalmente esta clase se extiende a lo largo y muy poco a lo ancho.

Tabla 5. Similitud entre los modelos probados y los mapas reales.

\begin{tabular}{|c|c|c|c|c|}
\hline \multicolumn{5}{|c|}{ Coeficiente de Dice } \\
\hline Modelo & Clase 1 & Clase 2 & Clase 3 & Clase 4 \\
\hline CNN mapa de entrenamiento & 0.561 & 0.864 & 0.526 & 0.230 \\
\hline CNN mapa de validación & 0.887 & 0.774 & 0.073 & 0.107 \\
\hline Distancia mínima con firmas espectrales entrenamiento & 0.576 & 0.819 & 0.593 & 0.054 \\
\hline Distancia mínima con firmas espectrales validación & 0.858 & 0.614 & 0.122 & 0.038 \\
\hline
\end{tabular}

Fuente: Autores. 
(a)

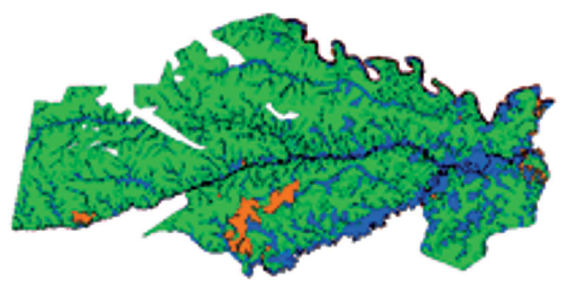

(b)

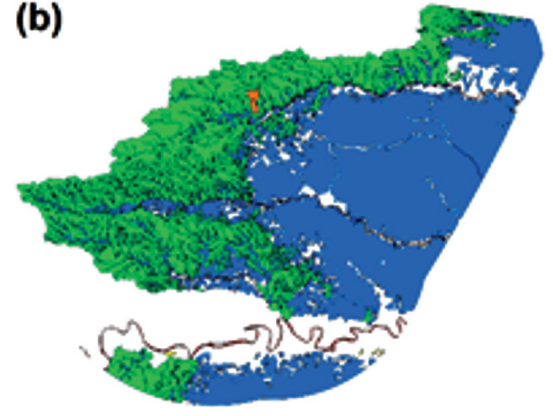

(d)

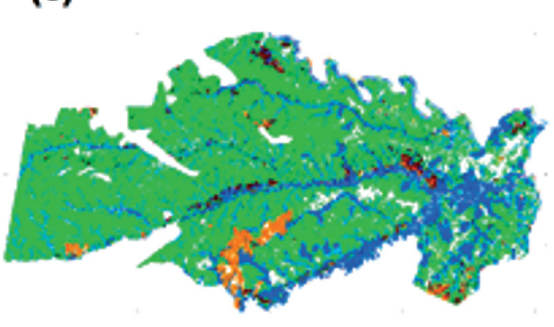

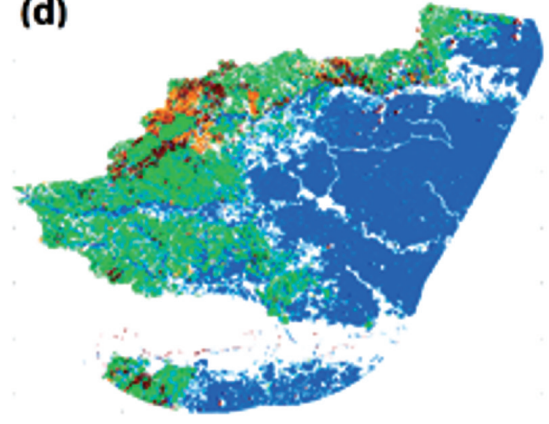

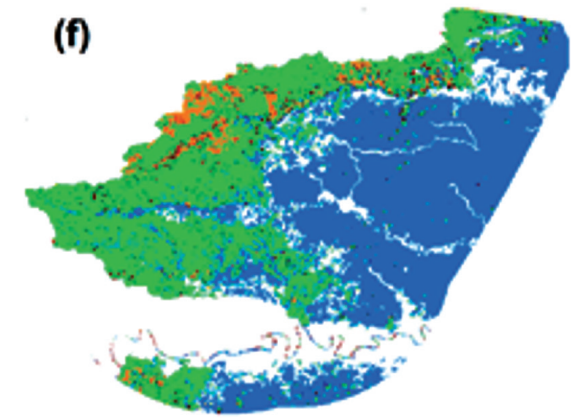

(e)

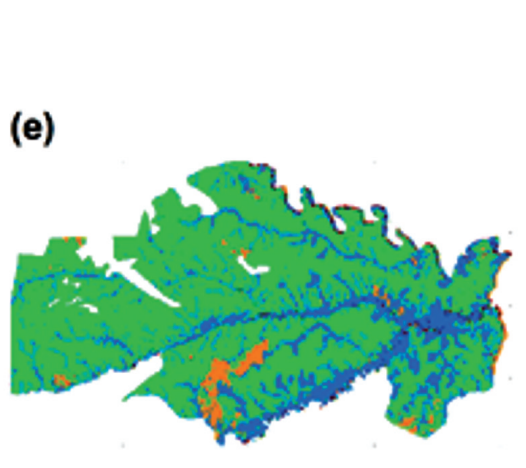

(c)
Clases

Leyenda

Bosques

Áreas con vegetación

herbácea y/o arbustiva

Áreas abiertas, sin o con poca vegetación

Áreas húmedas continentales
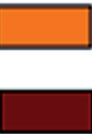

Figura 8. Comparación de mapas originales y los métodos computacionales usados para predecir mapas de cobertura con la CNN y con el procedimiento de firmas espectrales. Mapas utilizados en el estudio. a) Área de interés del mapa de coberturas del Amazonas 2007. b) mapa del Parque Nacional el Tuparro. Fuente: (Acevedo, 2012), (Axesnet S.a.S, 2012), c) y d) Mapas correspondientes a la clasificación usando firmas espectrales - algoritmo de distancia mínima, e) y f) Mapas correspondientes a la clasificación usando CNN. Fuente: Autores.

La CNN en trabajos anteriores ha demostrado buenos resultados en conjuntos de datos grandes (Krizhevsky et al., 2012), por lo tanto, una cantidad de datos de entrenamiento mayor tiene el potencial de poder mejorar la clasificación y así poder aumentar el promedio de exactitud a $91.02 \%$ en validación. Los resultados obtenidos presentan respuestas acertadas y muestran avances en la aplicación de las CNN en la clasificación de coberturas de suelo, comparados con trabajos previos de clasificación de coberturas donde se logró 91.8\% de precisión general (Eisavi et al., 2015)with various phenological patterns from satellite imagery, is a particularly challenging task. However, supplementary information, such as multitemporal data and/or land surface temperature (LST o un $95.10 \%$ de precisión global (Liu et al., 2013). Solo habiendo probado la arquitectura más básica de las CNN y variado unas cuantas variables de las muchas posibles en este problema, indica la posibilidad de lograr obtener mejores resultados continuando con la optimización de las técnicas de preprocesamiento y procesamiento empleadas en este estudio.

\section{Conclusiones}

Se logró entrenar un modelo de aprendizaje de redes neuronales convolucionales para la clasificación de co- 
berturas de la tierra capaz de distinguir entre 3 clases de bosques, áreas seminaturales y una clase de superficies de agua.

Se desarrolló un módulo para la extracción de parches que tiene métodos de lectura de imágenes y mapas de coberturas, con el cual se generó el modelo de entrenamiento y validación. Además, se generó un módulo de lectura y generación de objetos para el ingreso de lotes de imágenes y etiquetas de las clases a la CNN. Y por último un algoritmo de aprendizaje computacional de arquitectura ConvNet, el cual entrenó el modelo predictivo con un promedio de exactitud del $83.27 \%$ en entrenamiento y $91.02 \%$ en validación.

La resolución espacial y espectral es un factor determinante en la investigación de clasificación automática de cobertura del suelo. Se puede concluir que el procedimiento con CNN es capaz de clasificar satisfactoriamente coberturas de suelo, al compararlo con un método clásico de clasificación usando firmas espectrales mediante el algoritmo de distancia mínima.

Se planea trabajar con más arquitecturas de redes neuronales buscando lograr la clasificación de más clases de coberturas, además de realizar pruebas de validación de resultados mediante el uso de mapas de coberturas e imágenes de sensores remotos de otros estudios realizados en Colombia o a nivel internacional.

\section{Agradecimientos}

Se agradece a la Gobernación del Meta por el créditobeca condonable de la pasantía de Joven Investigador Arnol Sneider Suarez Londoño en la Universidad de los Llanos y a los grupos de investigación Macrypt y GITECX de la Universidad de los Llanos. Jiménez A. F. expresa su agradecimiento a la Gobernación de Boyacá, por la beca de doctorado de la convocatoria para la formación de capital humano de alto nivel para el departamento de Boyacá - 2015 y también a la Universidad de los Llanos. Cruz-Roa A. A. agradece parte del apoyo a los proyectos de investigación C05F02-038-2016 y C05-F02-039-2016.

\section{Referencias}

Acevedo L, Grupo de planeacion y manejo, S. D. G. Y. M. D. A. P. (2012). Parques nacionales naturales de colombia. Retrieved from http://mapas.parquesnacionales.gov.co/\#

Axesnet S.a.S. (2012). Sistema de Informacion Ambiental de Colombia - SIAC. Retrieved from http://www.siac.gov.co/Catalogo_mapas.html

Backoulou GF, Elliott NC, Giles KL, Mirik M. Processed multispectral imagery differentiates wheat crop stress caused by greenbug from other causes. Computers and Electronics in Agriculture. 2015;115:34-39. https://doi.org/10.1016/j.compag.2015.05.008

Bokusheva R, Kogan F, Vitkovskaya I, Conradt S, Batyrbayeva M. Satellite-based vegetation health indices as a criteria for insuring against drought-related yield losses. Agricultural and Forest Meteorology. 2016;220:200-206. https://doi.org/10.1016/j. agrformet.2015.12.066

Cruz-Roa A, Arévalo J, Judkins A, Madabhushi A, González F. 2015. A method for medulloblastoma tumor differentiation based on convolutional neural networks and transfer learning. International Symposium on Medical Information Processing and Analysis, 9681, 968103. https://doi.org/10.1117/12.2208825

Cruz-Roa A, Basavanhally A, González F, Gilmore H, Feldman M, Ganesan S, Madabhushi A. Automatic detection of invasive ductal carcinoma in whole slide images with convolutional neural networks. Proc. SPIE, 2014;9041(216):904103-904115. https://doi.org/10.1117/12.2043872

Eisavi V, Homayouni S, Yazdi AM, Alimohammadi A. Land cover mapping based on random forest classification of multitemporal spectral and thermal images. Environmental Monitoring and Assessment. 2015;187(5):1-14. https://doi.org/10.1007/ s10661-015-4489-3

Huang JT, Li J, Gong Y. 2015. An analysis of convolutional neural networks for speech recognition. In 2015 IEEE International Conference on Acoustics, Speech and Signal Processing (ICASSP) (pp. 4989-4993). IEEE. https://doi.org/10.1109/ ICASSP.2015.7178920

IDEAM, IGAC, \& CORMAGDALENA. (2008). Mapa de Cobertura de la Tierra Cuenca Magdalena-Cauca: Metodología CORINE Land Cover adaptada para Colombia a escala 1:100.000. Instituto de Hidrología, Meteorología y Estudios Ambientales, Instituto Geográfico Agustín Codazzi y Corporación Autónoma Regional del río Grande de la Magdalena (Vol. 1).

Krizhevsky A, Sutskever I, Hinton GE. 2012. ImageNet Classification with Deep Convolutional Neural Networks. Advances In Neural Information Processing Systems. 1-9. https://doi.org/http:// dx.doi.org/10.1016/j.protcy.2014.09.007

Liu Y, Zhang B, Wang LM, Wang N. A self-trained semisupervised SVM approach to the remote sensing land cover classification. Computers and Geosciences. 2013;59:98-107. https://doi. org/10.1016/j.cageo.2013.03.024

Martin M, Newman S, Aber J, Congalton R. Determining forest species composition using high spectral resolution remote sensing data. Remote Sensing of Environment. 1998;65(3):249-254. https://doi.org/10.1016/S0034-4257(98)00035-2

Ministerio del Medio Ambiente. (2010). Leyenda nacional de coberturas de la tierra.

Perlin HA, Lopes HS. Extracting human attributes using a convolutional neural network approach. Pattern Recognition Letters. 2015;68:250-259. https://doi.org/10.1016/j. patrec.2015.07.012

Rodriguez-Galiano VF, Ghimire B, Rogan J, Chica-Olmo M, RigolSanchez JP. An assessment of the effectiveness of a random forest classifier for land-cover classification. ISPRS Journal of 
Photogrammetry and Remote Sensing. 2012;(67):93-104. https://doi.org/10.1016/j.isprsjprs.2011.11.002

Rujoiu-Mare MR, Mihai B. Mapping Land Cover Using Remote Sensing Data and GIS Techniques: A Case Study of Prahova Subcarpathians. Procedia Environmental Sciences. 2016;32:244-255. https://doi.org/10.1016/j.proenv.2016.03.029

Thonfeld F, Feilhauer H, Braun M, Menz G. Robust Change Vector Analysis (RCVA) for multi-sensor very high resolution optical satellite data. International Journal of Applied Earth Observation and Geoinformation. 2016;50:131-140. https://doi. org/10.1016/j.jag.2016.03.009
Wang H, Cruz-Roa A, Basavanhally A, Gilmore H, Shih N, Feldman $M$, Madabhushi A. Mitosis detection in breast cancer pathology images by combining handcrafted and convolutional neural network features. Journal of Medical Imaging (Bellingham, Wash.). 2014;1(3):34003. https://doi.org/10.1117/1.JMI.1.3.034003

Warner TA, Foody GM, Nellis MD. 2009. The SAGE Handbook of Remote Sensing. 504. https://doi.org/10.4135/9780857021052

Zhang R, Zhu D. Study of land cover classification based on knowledge rules using high-resolution remote sensing images. Expert Systems with Applications, 2011;38(4):3647-3652. https://doi. org/10.1016/j.eswa.2010.09.019 